Research Article

Cellular, Molecular and Developmental Genetics

\title{
Overexpression of RAPGEF3 enhances the therapeutic effect of dezocine in treatment of neuropathic pain
}

\author{
Xue Liu ${ }^{1}$, Li Song ${ }^{1}$, Xiaojun $\mathrm{Ma}^{1}$, Yong $\mathrm{Liu}^{1}$, Hui Huang ${ }^{1}$, Yongsheng $\mathrm{Xu}^{1}$ and Wei Yan ${ }^{1}$ (D) \\ ${ }^{1}$ The Affiliated Hospital of Qingdao University, Department of Anesthesiology, Qingdao, Shandong, China.
}

\begin{abstract}
Pain is a significant problem worldwide that affects the quality of life of patients. Dezocine is a non-addictive analgesic drug with kappa-opioid antagonist activity and has been successfully used to alleviate of postoperative pain. In addition, dezocine has an analgesic effect similar to that of morphine, alleviating moderate to severe pain. Rap guanine nucleotide exchange factor 3 (RAPGEF3) is a guanine nucleotide exchange factor for GTPases Rap1 and Rap2, which could enhance the activity of Rap 1 to promote cell adhesion and axon regeneration, as well as promote neurite extension by interacting with nerve growth factors. Here, we first observed that overexpression of RAPGEF3 increased cell viability, as shown by a CCK-8 assay, and recovered brain function in rats. The expression of inflammation-related factors at the mRNA level was detected using $\mathrm{qPCR}$, and the concentration of these factors in a cultured cell medium and rat serum samples were decreased as shown by ELISA after RAPGEF3 overexpression. Through western blotting, we further found that pro-inflammatory proteins were decreased, and these effects might be mediated by inhibition of the Ras/p-38 MAPK signaling pathway. Taken together, we speculated that RAPGEF3overexpression enhances the therapeutic effect of dezocine on neuropathic pain by inhibiting the inflammatory response through inhibition of the Ras/p-38 MAPK signaling pathway.
\end{abstract}

Keywords: RAPGEF3, dezocine, neuropathic pain, Ras/p-38 MAPK, inflammation.

Received: December 15, 2020; Accepted: August 05, 2021.

\section{Introduction}

Neuropathic pain (NP) is normally caused by lesions in the nervous system and leads to long-term debilitating conditions, which reduce the quality of life. Pathophysiological changes in patients with NP result in anxiety, depression, and elevated blood pressure (Kroth et al., 2020). There are several types of NP, including painful non-diabetic neuropathy, trigeminal neuralgia, multiple sclerosis, and spinal cord injury (Bouhassira, 2019). NP is a commonly encountered disease, but effective treatment for patients with NP still lacks due to the adverse effects of treatment drugs. Dezocine, also known as a partial $\mu$ opioid receptor agonist and $\kappa$ receptor agonist, is a new narcotic analgesic that has been widely used to treat anesthesia analgesia, with clinical trials to treat neuropathic pain having been conducted (Zhang et al., 2019). Dezocine has been used to reduce postoperative pain after surgery at a regular dose of 5-10 mg (Pandit et al., 1986), and its effect in treating moderate to severe pain has been considered approximately equal to that of morphine, without a tolerance effect (Walker et al., 1999). A previous study found that the combination of dezocine and midazolam has a treatment effect in relieving pain and anxiety in patients (Walker et al., 1999). Other studies showed that dezocine antagonizes morphine analgesia in acute nociception (Li et al., 2017), and could be successfully applied to anesthetize of indolent colonoscopy patients without pain in combination with propofol (Bos, 2006). Cyclic adenosine monophosphate

Send correspondence to Wei Yan, Hospital of Qingdao University, Department of Anesthesiology, Qingdao, Shandong, China. E-mail: doc_xu2018@163.com.
(cAMP) is a well-known regulator of biological processes, including gene transcription, cell proliferation, migration and apoptosis. Rap guanine nucleotide exchange factor 3 (RAPGEF3) was first identified in 1998 as a new cAMP sensor of cAMP (Bramanti et al., 2015), and has been regarded as an important regulator of many cellular processes, such as survival, proliferation and neuronal signaling (Xu et al., 2016a). Using a RAPGEF3 deletion mouse model, researchers observed increased expression of RAPGEF3 in the root ganglia (DRG) after nerve transection, reducing the inflammatory mediator as well as NLRP3 inflammasome (Carswell et al., 1975). In addition, overexpression of RAPGEF3 has been found to promote the neointimal formation in aortic tissues (Chaplan et al., 1994). RAPGEF3 regulates inflammation and plays a critical role in inflammatory pain through the ERK signaling pathway, and has been regarded as a therapeutic target for chronic pain (Pan et al., 2019). However, the function of RAPGEF3 in NP remains unclear.

\section{Material and Methods}

\section{Reagents}

H-DMEM (11965092) and FBS (10093) were purchased from Thermo Fisher Scientific (Waltham, MA, USA). BsmBI (R0739S), KpnI (R3142S), and BamHI (R3136S) were purchased from Mew England Biolabs (Ipswich, MA, USA). Dezocine was acquired from the Dezocine Yangtze River Pharmaceutical Group (Jiangsu, China). TNF- $\alpha$ (H8916), IL$1 \beta$ (SRP6169), CCL2 (MSST0036), and CXCL1 (SRP3216) were purchased from Sigma-Aldrich (St Louis, MO, USA). Anti-SOCS3 (ab16030), Epac1 (ab21236), GRK2 (ab137666), PDE4B (ab14611), PACAP (ab181205), PKC $\alpha$ (ab32376), 
p38 (ab31828), p38 (phospho T180 + Y182) (ab4822), Ras (ab180772), Raf(ab137435), RAP1(ab113480) antibody and IL-1 $\beta$ (ab197742), IL-6 (ab100713), TNF- $\alpha$ (ab208348) and COX-2 (ab210574) ELISA kits were purchased from Abcam (Cambridge, UK).

\section{Cell culture}

Primary glial cells were acquired previously described (Han et al., 2018). The cerebral cortex was obtained from newborn SD rats (2-4 days old, housed under 50-60\% humidity, $22-24{ }^{\circ} \mathrm{C}$ atmosphere with a $12 \mathrm{~h} \mathrm{light/dark}$ cycle, water and food freely available), and the cerebral cortex was removed and digested using $0.25 \%$ trypsin (supplied with $0.4 \%$ DNase). Digested glial cells and 293 T cells were cultured in an H-DMEM medium with $10 \%$ FBS under a humidified $37{ }^{\circ} \mathrm{C}$ atmosphere supplied with $5 \% \mathrm{CO}_{2}$. After 2 days of culture, the medium was changed to remove unattached cells.

\section{Vector construction}

The full-length $R A P G E F 3$ gene was acquired using PCR with the following primers: Forward: 5'-CCGAAGCT GCTCCTACCA-3', Reverse: 5'-ACTCCTCGCTGTTGGTG AGT-3' (reaction steps: $50^{\circ} \mathrm{C}$ for $30 \mathrm{~min}$ and $85^{\circ} \mathrm{C}$ for $5 \mathrm{~min}$. Amplified size: $594 \mathrm{bp}$ ). Thereafter, the PCR products and pcDNA3.1 blank vector were digested with KpnI and BamHI, and linked with DNA ligase. Next, the pcDNA3.1-RAPGEF3 overexpression vector was transfected into primary glial cells using Lipofectamine 2000 Transfection Reagent for $48 \mathrm{~h}$, and the stably expressed cells were screened using G418. The $R A P G E F 3$ knockdown vector was constructed according to a previous study (Liu et al., 2017) with the following pair of primer: Forward: 5'-CAGAGAGGCGGCGATGCCAC-3', Reverse: 5'-CAGAGAGGCGGCGATGCCAC-3'. Briefly, oligos were first annealed following the instructions, and blank vectors of lenti-CRISPR v2 and annealed oligos were digested with BsmBI for $30 \mathrm{~min}$ at $37^{\circ} \mathrm{C}$. After linking with T4 PNK, the RAPGEF3 knockdown vector was transfected into 293 T cells to construct $R A P G E F 3$ knockdown lentivirus to transfect primary glial cells, and the stably expressed cells were screened using $2 \mu \mathrm{g} / \mathrm{mL}$ puro.

\section{CCK-8 assay}

The CCK-8 assay was performed according to the manufacturer's instructions (CA1210; Solarbio Life Sciences, Beijing, China). Briefly, cells were seeded into each well of a 96-well plate at a concentration of $1 \times 10^{4}$, and the cells were grouped and treated as previously described. Cells were then incubated with CCK- 8 reagent for $4 \mathrm{~h}$, and the OD value at $450 \mathrm{~nm}$ was detected using a SpectraMax iD3 microplate reader (Molecular Devices, San Jose, CA, USA).

\section{Model construction}

After the confluence of glial cells reached $70-80 \%$, cells were treated with TNF- $\alpha(10 \mathrm{ng} / \mathrm{mL}), \mathrm{IL}-1 \beta(10 \mathrm{ng} / \mathrm{mL})$, CCL2 (100 ng/mL), and CXCL1 (312 ng/mL) to mimic the inflammatory conditions of neuropathic pain before performing subsquent experiments. The cells were then divided into four groups: normal group (NC), dezocine treatment group (DT), dezocine treatment combined with RAPGEF3 overexpression group (DO), and dezocine treatment combined with RAPGEF3 inhibition group (DI). In the dezocine treated group, the cells were treated with $9 \mathrm{ng} / \mathrm{mL}$ dezocine for 10 consecutive days. Ten SD rats, five RAPGEF3 overexpression and five RAPGEF3 knockdown rats were purchased from Cyagen (Santa Clara, CA, USA). Rats were kept in a $22-24{ }^{\circ} \mathrm{C}, 50-60 \%$ humidity atmosphere with a 12-h light/dark cycle, with freely available water and food. The neuropathic pain after sciatic nerve injury model was constructed according to a previous study (Ding et al., 2017). Briefly, rats were first anesthetized with isoflurane, and then the left sciatic nerve was loosely ligated using 4-0 chromic gut sutures at four segments $1 \mathrm{~mm}$ apart. Next, the sutures were tightened gently until a brisk twitch of the left hind limb was observed. The same procedure was performed on the rats in the sham group except for sciatic nerve ligation. Rats were divided into four groups: sham group (SH), dezocine treatment group (TD), dezocine treatment combined with $R A P G E F 3$ overexpression group (TO), and dezocine treatment combined with $R A P G E F 3$ inhibition group (TI). In the dezocine treated group, rats were injected with dezocine via intraperitoneal injection at a concentration of 3 $\mathrm{mg} / \mathrm{kg} 30 \mathrm{~min}$ before the procedure.

\section{Behavioral testing}

Mechanical allodynia in rats was detected using von Frey filaments. Rats in each group were divided and treated as previously described, and then the rats were placed in transparent Plexiglas compartments on a metal mesh. The surface of the left paw was subjected to von Frey hairs with logarithmically increasing stiffness (0.4-26 g). Rapid withdrawal or flinching of the paw was considered a positive response. Thereafter, the 50\% paw withdrawal threshold (PWT) was detected using Dixon's up-down method (Yokoyama et al., 2008). Thermal hyperalgesia was detected using a Hargreaves apparatus with radiant heat and presented as paw-withdrawal latency (PWL). Briefly, rats were placed in hyaline plastic compartments, while the left hind paw was exposed to a source of radiant heat via a glass plate within $20 \mathrm{~s}$.

\section{Ethical statement}

The experiments were performed in accordance with the guidelines of the ethics committee of the Affiliated Hospital of Qingdao University (Qingdao, Shandong, No.SCNUSLS-2017-03), and all animals were treated under the guidelines of the Public Health Service Policy on the Humane Care and Use of Laboratory Animals of the National Institutes of Health.

\section{RNA extraction}

Rats and glial cells were grouped and treated as previously described, and the RNA extraction process was performed according to the protocol of the Total RNA Extraction Kit (R1200, Solarbio Life Sciences). Briefly, the cells and brain tissues of rats were lysed with lysis buffer, followed by incubation at room temperature for $5 \mathrm{~min}$. After incubation with chloroform, the samples were centrifuged at $12,000 \mathrm{rpm}$ for $5 \mathrm{~min}\left(4^{\circ} \mathrm{C}\right)$. The water phase of the samples was then removed into the absorption tube and centrifuged at $12,000 \mathrm{rpm}$ for $2 \mathrm{~min}$, washed with washing buffer, and then eluted using elution buffer and stored at $-80{ }^{\circ} \mathrm{C}$ until subsequent experiments. The quality and integrity of RNA 
samples were determined using agarose gel electrophoresis. If the $28 \mathrm{~S}$ and $18 \mathrm{~S}$ bands were clearly observed in the sample and the intensity of the $28 \mathrm{~S}$ band was more than 2-fold that of the $18 \mathrm{~S}$ band, we considered the quality and integrity of RNA samples as acceptable for further experiments.

\section{Reverse transcription and quantitative real-time polymerase chain reaction (qPCR)}

Reverse transcription and qPCR were performed using the UltraSYBR One-Step RT-qPCR Kit according to the manufacturer's instructions (CW0659, CWBio, Beijing, China). Briefly, the reaction mixture was prepared as recommended, and the primers used were as follows: $I L-1 b$ : Forward: 5'-GACTTCACCATGGAACCCGT-3', reverse: 5'-GGAGACTGCCCATTCTCGAC-3'; IL-6: Forward: 5'-ACCCCAACTTCCAATGCTCT-3', reverse: 5'-GGTTTGCCGAGTAGACCTC-3'; TNFA: Forward: 5'-AACACACGAGACGCTGAAGT-3', reverse: 5'-TC CAGTGAGTTCCGAAAGCC-3'; PTGS2: Forward: 5'-CAC GGACTTGCTCACTTTGTT-3', reverse: 5'-AAGCGTTTG CGGTACTCATT-3'. The reaction was performed using the following steps: reverse transcription at $45^{\circ} \mathrm{C}$ for $10 \mathrm{~min}$, pre-degeneration at $95^{\circ} \mathrm{C}$ for $5 \mathrm{~min}$. Subsequently, the following steps were repeated for 36 cycles: denaturation at $95{ }^{\circ} \mathrm{C}$ for $15 \mathrm{~s}$, annealing at $58^{\circ} \mathrm{C}$ for $30 \mathrm{~s}$ and extension at $72{ }^{\circ} \mathrm{C}$ for 30 $\mathrm{s}$. Each experiment was replicated thrice independently, and GAPDH was used as an internal control. The expression of each gene was determined using the $2^{-\Delta \Delta \mathrm{Cq}}$ method (Grimholt et al., 2013).

\section{Protein collection and western blot analysis}

The cells and brain tissues were cultured and grouped as previously described. After treatment, cells and brain tissues were lysed with lysis buffer ( $8 \mathrm{M}$ Urea, $50 \mathrm{mM}$ IAA, $10 \mathrm{mM}$ DTT and a proteinase inhibitor cocktail). After centrifugation, the samples were collected into a new tube, and the concentration of proteins was determined using the BCA assay. Protein samples $(60 \mu \mathrm{g})$ were loaded and separated by $10 \%$ SDS-PAGE, and proteins were transferred onto nitrocellulose membranes. Membranes were then incubated with $5 \%$ skim milk for $1 \mathrm{~h}$ at room temperature, followed by incubation with primary antibodies at $4{ }^{\circ} \mathrm{C}$ overnight. After incubation with a secondary antibody for $1 \mathrm{~h}$ at room temperature, the gray value of proteins was determined using a chemiluminescent immunoassay, and the expression of each target protein was measured using IPP 6.0.

\section{Enzyme-linked immunosorbent assay (ELISA)}

ELISA was performed according to the ELISA kit protocol. Briefly, serum samples and cultured cell media were added into each well of a 96-well plate, and the samples were incubated with an antibody at room temperature for $1 \mathrm{~h}$. Afterward, samples were washed with washing buffer thrice, incubated with TMB substrate for $10 \mathrm{~min}$, and the OD value at $450 \mathrm{~nm}$ was measured using a SpectraMax iD3 microplate reader (Molecular Devices).

\section{Statistical analysis}

Data of these experiments are presented as mean \pm SEM. Each experiment was replicated thrice independently.
Differences between groups were analyzed using one-way ANOVA in SPSS (version 22.0, SPSS Inc, Chicago, IL, USA). Statistical significance was set at $P<0.05$.

\section{Results}

\section{Detection of cell viability in each group using the CCK-8 assay}

As shown in Figure 1A, the viability rate was significantly increased in the DT and DO groups $(\mathrm{P}<0.05)$ compared with that in the NC group, significantly increased in the DO group $(\mathrm{P}<0.05)$ and significantly decreased in the DI group $(\mathrm{P}<0.05)$ compared with that in the DT group. Therefore, the results showed that dezocine increased the viability rate of cells, and overexpression of RAPGEF3 enhanced this effect.

\section{Western blotting analysis of RAPGEF3 expression in each group of cell and rat models}

As shown in Figures $1 \mathrm{~B}$ and $1 \mathrm{C}$, the expression of RAPGEF3 was significantly increased in the overexpression group and significantly decreased in the inhibition group $(\mathrm{P}<0.05)$, indicating that the RAPGEF3 overexpression and inhibition model was successfully constructed in cells and rats.

The effect of overexpression of RAPGEF3 combined with dezocine on the cognitive performance of rats in the acquisition trial and MWM probe test

As shown in Figure 2, the performance of rats in each group in the MWM test is presented. The escape latency was significantly reduced in the TO group $(\mathrm{P}<0.05)$, and the average number of crossing platforms and the time spent in the target quadrant were significantly higher in the TO group than those in the SH and TD groups. These results indicate that dezocine treatment enhances memory function, and overexpression of RAPGEF3 enhances this trend.

\section{qPCR analysis of inflammation-related molecule expression for each group in cell and rats models}

As shown in Figures 3 and 4, the expression of $I L-1 b$, $I L-6, T N F A$ and PTGS2 was determined using qPCR. The fold change of $I L-1 b$ was $1.00 \pm 0.08,0.70 \pm 0.06,0.40 \pm$ 0.03 , and $0.76 \pm 0.06$ in the $\mathrm{NC}, \mathrm{DT}, \mathrm{DO}$, and DI groups, respectively, and was significantly decreased in all treatment groups relative to that in the $\mathrm{NC}$ group $(\mathrm{P}<0.05)$, and in the DO group compared with that in the DT group $(\mathrm{P}<0.05)$ in the cell model. The fold change of $I L-1 b$ was $1.00 \pm 0.07$, $0.87 \pm 0.07,0.48 \pm 0.02$, and $1.00 \pm 0.07$ was significantly decreased in the TO group compared with the SH and TD groups $(\mathrm{P}<0.05)$ in the rat model. The fold change of $I L-6$ was $1.00 \pm 0.07,0.85 \pm 0.06,0.51 \pm 0.04$, and $0.97 \pm 0.07$, and was significantly decreased in the DO group compared with that in the $\mathrm{NC}$ and DT groups $(\mathrm{P}<0.05)$. The fold change of $I L-6$ was $1.00 \pm 0.08,0.77 \pm 0.06,0.53 \pm 0.04$, and $0.88 \pm$ 0.07 and was significantly decreased in the TD and TO groups compared with that in the SH group $(\mathrm{P}<0.05)$ in cell model, as well as in the TO group compared with that in the TD group $(\mathrm{P}<0.05)$ in the rat model. The fold change of $T N F-A$ was $1.00 \pm 0.08,0.80 \pm 0.06,0.55 \pm 0.04$ and $0.89 \pm 0.07$, respectively, and was significantly decreased in the DT and DO groups than that in the $\mathrm{NC}$ group $(\mathrm{P}<0.05)$, as well as in 
the DO group compared with that in the DT group $(\mathrm{P}<0.05)$ in the cell model. The fold change of $T N F-A$ was $1.00 \pm 0.07$, $0.87 \pm 0.07,0.59 \pm 0.05$, and $0.95 \pm 0.07$, respectively, and was significantly decreased in the TO group relative to that in the $\mathrm{SH}$ and TD groups $(\mathrm{P}<0.05)$ in the rat model. The fold change of $P T G S 2$ was $1.00 \pm 0.08,0.79 \pm 0.06,0.53 \pm 0.04$, and $0.95 \pm 0.08$, and was significantly decreased in the DT and DO groups compared with that in the $\mathrm{NC}$ group $(\mathrm{P}<0.05)$, significantly decreased in the DO group and significantly increased in the DI group than that in the DT group $(\mathrm{P}<0.05)$ in the cell model. The fold change of PTGS2 was $1.00 \pm 0.07$, $0.83 \pm 0.07,0.62 \pm 0.05$, and $0.96 \pm 0.08$, and was significantly decreased in the DT and DO groups compared with that in the SH groups $(\mathrm{P}<0.05)$, as well as in the DO group compared with that in the DT group $(\mathrm{P}<0.05)$.

\section{Western blotting analysis of inflammation-related proteins in each group of cells and the rat model}

As shown in Figures 5 and 6, the expression of inflammation-related proteins in cell and rat models was determined using western blotting analysis. Briefly, the expression of SOCS3 was significantly decreased in the DT and
DO groups than that in the NC group $(\mathrm{P}<0.05)$, significantly decreased in the DO group and significantly increased in the DI group relative to that in the DT group $(\mathrm{P}<0.05)$ in the cell model, whereas it was significantly decreased in the DO group compared with that in the SH and DT groups $(\mathrm{P}<0.05)$ in the rat model. The expression of GRK2 was significantly increased in all treatment groups than that in the $\mathrm{NC}$ group $(\mathrm{P}<0.05)$ in the cell model, significantly increased in the DO group, and significantly decreased in the DI group compared with that in the DT group $(\mathrm{P}<0.05)$ in the cell model. Additionally, GRK2 expressoin was significantly increased in the TD and TO groups and significantly decreased in the TI group relative to that in the $\mathrm{SH}$ group $(\mathrm{P}<0.05)$, significantly increased in the TO group, and significantly decreased in the TI group compared with that in the TD group $(\mathrm{P}<0.05)$ in the rat model. The expression of PDE4B was significantly decreased in the DO group and significantly increased in the DI group compared with that in the $\mathrm{NC}$ and DT groups $(\mathrm{P}<0.05)$ in the cell model and was significantly decreased in the TO group compared with that in the SH and TD groups $(\mathrm{P}<0.05)$ in the rat model. The expression of PACAP was significantly higher in the DO group than that in the $\mathrm{NC}$ and DT groups $(\mathrm{P}<0.05)$ in
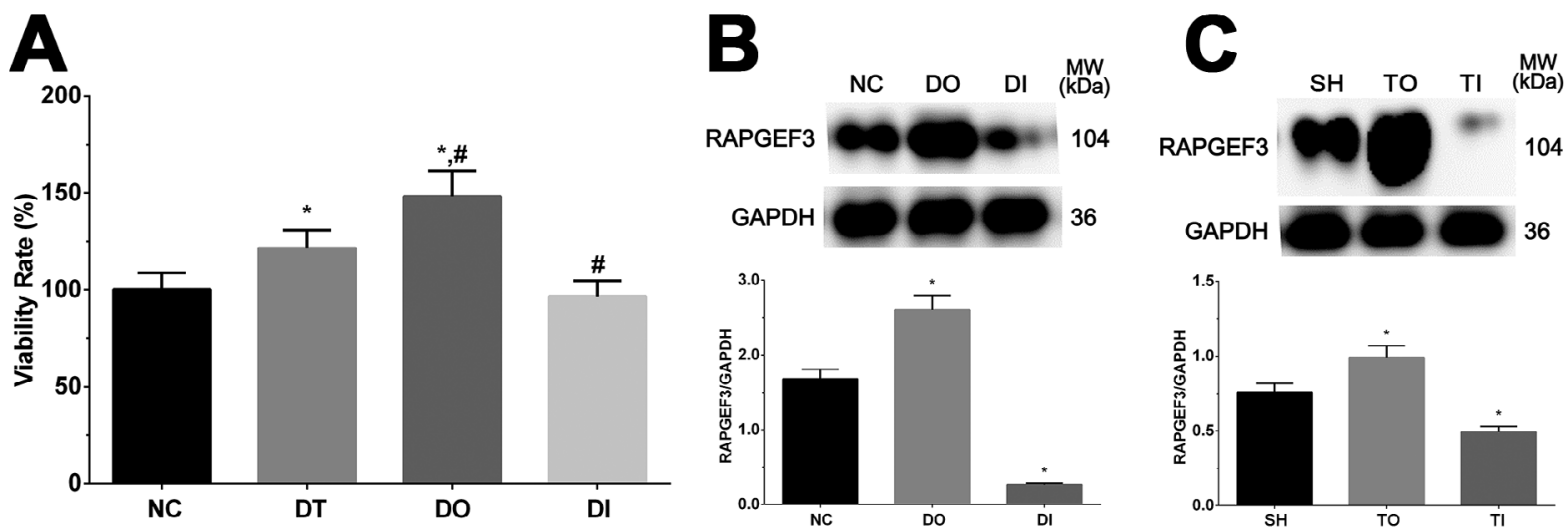

Figure 1 - Viability rate of glial cells in NC, DT, DO and DI group. (A) Viability rate of glial cells. (B) Expression of RAPGEF3 in NC, DO and DI group of glial cells without treatment of dezocine. (C) Expression of RAPGEF3 in SH, TO and TI group of rats model without treatment of dezocine. Data was presented as mean $\pm \mathrm{SD}$, each experiment was repeated for three times. ${ }^{*} \mathrm{P}<0.05$ vs $\mathrm{NC}$ group of cells or $\mathrm{SH}$ group of rats. \#P<0.05 vs signal dezocine treatment group. NC: normal group, DT: dezocine treatment group, DO: dezocine treatment combined with RAPGEF3 overexpression group, DI: dezocine treatment combined with RAPGEF3 inhibition group. SH: sham group, TD: dezocine treatment group, TO: dezocine treatment combined with RAPGEF3 overexpression group, TI: dezocine treatment combined with RAPGEF3 inhibition group.
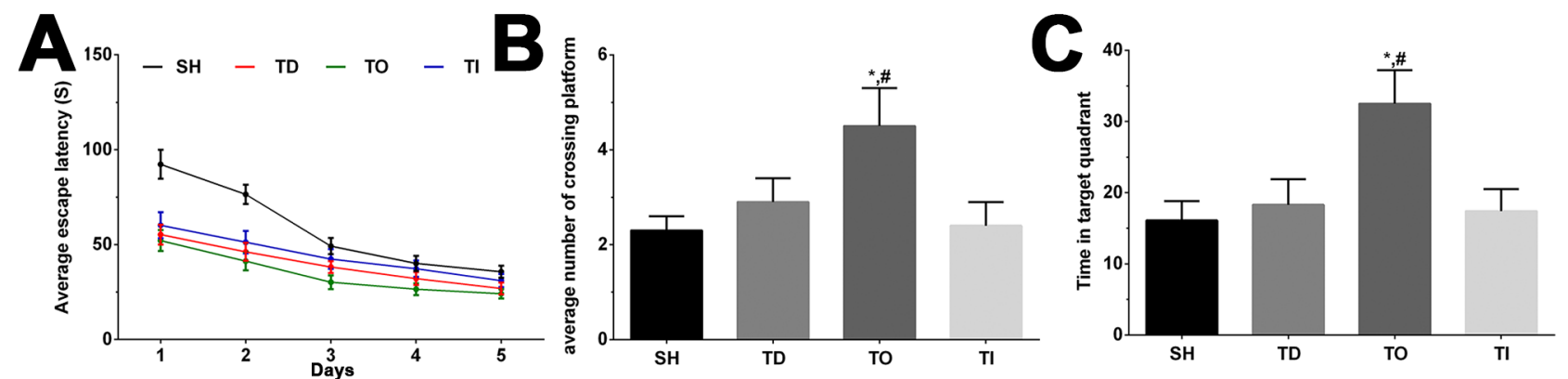

Figure 2 - Detection of memory function using MWM. (A) The escape latency of rats in each group in a continuous 5 days. (B) The average number of crossing platform of rats in each group. (C) The time in target quadrant of rats in each group. Data was presented as mean \pm SD. Each experiment was repeated for three times independently. ${ }^{*} \mathrm{P}<0.05$ compared with $\mathrm{SH}$ group. $\# \mathrm{P}<0.05$ compared with TD group. NC: normal group, DT: dezocine treatment group, DO: dezocine treatment combined with RAPGEF3 overexpression group, DI: dezocine treatment combined with RAPGEF3 inhibition group. SH: sham group, TD: dezocine treatment group, TO: dezocine treatment combined with RAPGEF3 overexpression group, TI: dezocine treatment combined with RAPGEF3 inhibition group. 

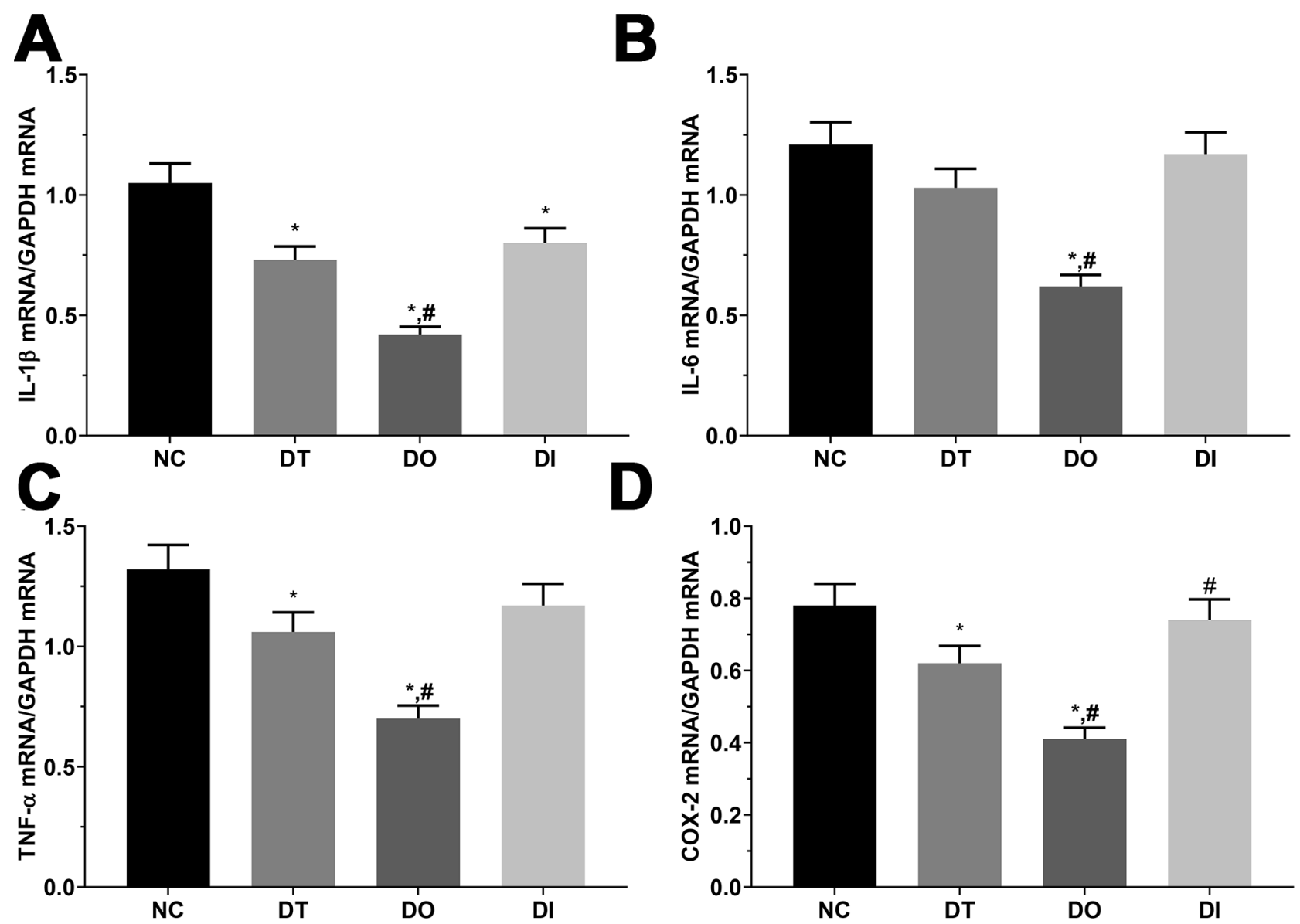

Figure 3 - Detection of pro-inflammatory factors in glial cells. (A) Expression of $I L$ - $1 b$ mRNA in NC, DT, DO and DI group of glial cells. (B) Expression of $I L-6$ mRNA in NC, DT, DO and DI group of glial cells. (C) Expression of TNF-A in NC, DT, DO and DI group of glial cells. (D) Expression of PTGS2 in NC, DT, DO and DI group of glial cells. Data was presented as mean $\pm \mathrm{SD}$, each experiment was repeated for three times. $* \mathrm{P}<0.05$ vs $\mathrm{NC}$ group of cells. \#P $<0.05$ vs signal dezocine treatment group. NC: normal group, DT: dezocine treatment group, DO: dezocine treatment combined with RAPGEF3 overexpression group, DI: dezocine treatment combined with RAPGEF3 inhibition group. SH: sham group, TD: dezocine treatment group, TO: dezocine treatment combined with RAPGEF3 overexpression group, TI: dezocine treatment combined with RAPGEF3 inhibition group.

the cell model and was significantly higher in the TO group than that in the SH and TD groups $(\mathrm{P}<0.05)$ in the rat model. The expression of PKC was significantly decreased in the DO group and significantly increased in the DI group compared with that in the NC and DT groups $(\mathrm{P}<0.05)$ in the cell model, and was significantly lower in the TO group than that in the $\mathrm{SH}$ and TD groups $(\mathrm{P}<0.05)$ in the rat model.

\section{Determination of Ras/MAPK signaling pathway activation in cell and rat models using western blotting analysis}

As shown in Figures 7 and 8, the activation of the Ras/MAPK signaling pathway in cell and rat models was determined using western blot analysis. The expression of RAPGEF3 was significantly increased in all treatment groups compared with that in the NC group $(\mathrm{P}<0.05)$, significantly increased in the DO group, and significantly decreased in the DI group relative to that in the DT group $(\mathrm{P}<0.05)$ in the cell model. Furthermore, RAPGEF3 expression was significantly increased in the TD and TO groups compared with that in the $\mathrm{SH}$ group $(\mathrm{P}<0.05)$, significantly increased in the TO group and significantly decreased in the TI group compared with that in the TD group $(\mathrm{P}<0.05)$ in the rat model. The expression of Ras was significantly decreased in the DT and DO groups compared with that in the $\mathrm{NC}$ group $(\mathrm{P}<0.05)$ and significantly decreased in the DO group compared with that in the DT group $(\mathrm{P}<0.05)$ in the cell model. Additionally, Ras expression was significantly decreased in the TO group than that in the $\mathrm{SH}$ and TD groups $(\mathrm{P}<0.05)$ and significantly increased in the TI group than that in the TD group $(\mathrm{P}<0.05)$ in the rat model. The expression of Raf was significantly decreased in the DO group compared with that in the $\mathrm{NC}$ and $\mathrm{DT}$ groups $(\mathrm{P}<0.05)$ in the cell model, as well as in the TD and TO groups and significantly increased in the TI group compared with that in the $\mathrm{SH}$ group $(\mathrm{P}<0.05)$. Furthermore, Raf expression was significantly decreased in the TO group and significantly increased in the TI group compared with that in the TD group $(\mathrm{P}<0.05)$ in the rat model. The expression of RAP1 was significantly increased in the DO group compared with that in the NC and DT groups and significantly decreased in the DI group compared with that in the DT group $(\mathrm{P}<0.05)$ in the cell model. Furthermore, RAP1 expression was significantly increased in all treatment groups than that in the $\mathrm{SH}$ group and significantly increased in the TO group than that in the 
TD group $(\mathrm{P}<0.05)$ in the rat model. The ratio of $\mathrm{p}-\mathrm{p} 38$ MAPK/p-38 MAPK was significantly decreased in the DO group and significantly increased in the DI group compared with that in the NC and DT groups $(\mathrm{P}<0.05)$ in the cell model. Additionally, the ratio of p-p38 MAPK/p-38 MAPK was significantly decreased in all treatment groups compared with that in the SH group $(\mathrm{P}<0.05)$, significantly decreased in the TO group, and significantly increased in the TI group relative to that in the TD group $(\mathrm{P}<0.05)$ in the rat model.

\section{Determination of the concentration of inflammatory cytokines in cultured cell media and serum samples of rats}

As shown in Figures 9 and 10, the concentration of IL- $1 \beta$ was significantly decreased in the DT and DO groups compared with that in the $\mathrm{NC}$ group $(\mathrm{P}<0.05)$, significantly decreased in the DO group, and significantly increased in the DI group compared with that in the DT group $(\mathrm{P}<0.05)$ in the cell model. In addition, IL- $1 \beta$ concentration was significantly decreased in the TD and TO groups than that in the SH group and significantly decreased in the TO group than that in the TD group $(\mathrm{P}<0.05)$ in the rat model. The concentration of IL-6 was significantly lower in the DT and DO groups than that in the NC group, and significantly decreased in the DO group compared with that in the DT group $(\mathrm{P}<0.05)$ in the cell model, and presented a similar trend in the rat model. The concentration of TNF- $\alpha$ was significantly lower in the DO group than that in the NC and DT groups $(\mathrm{P}<0.05)$ in the cell model. Furthermore, TNF- $\alpha$ concentration was significantly decreased in the TD and TO groups compared with that in the SH group $(\mathrm{P}<0.05)$, and significantly decreased in the TO group compared with that in the TD group $(\mathrm{P}<0.05)$ in the rat model. The concentration of COX-2 was significantly decreased in the DT and DO groups compared with that in the $\mathrm{NC}$ group $(\mathrm{P}<0.05)$, significantly decreased in the DO group and significantly increased in the DI group compared with that in the DT group $(\mathrm{P}<0.05)$ in the cell model. Furthermore, COX-2 concentration was significantly decreased in all treatment groups than that in the SH group and significantly decreased in the TO group relative to that in the TD group $(\mathrm{P}<0.05)$ in the rat model.
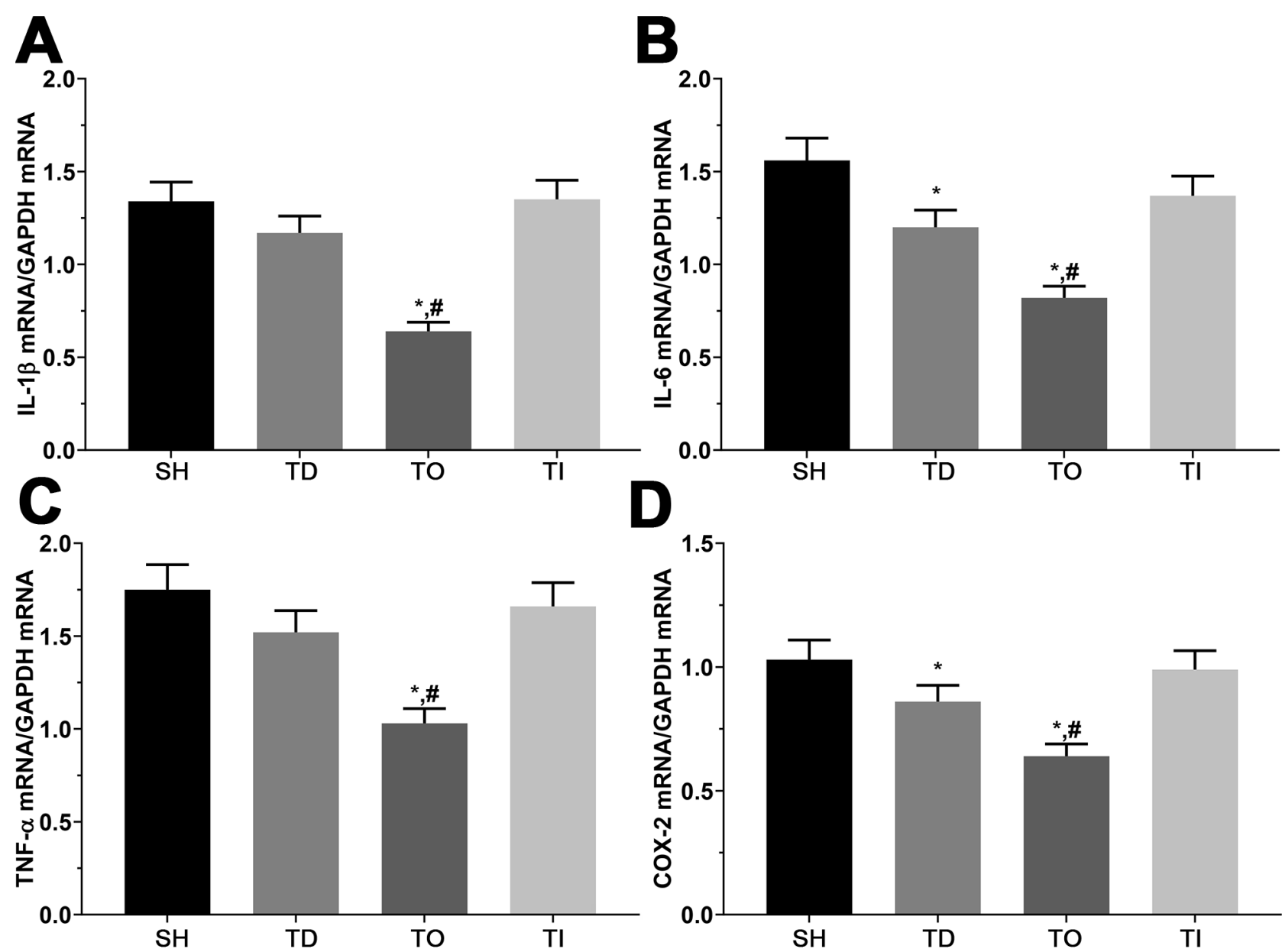

Figure 4 - Detection of pro-inflammatory factors in brain tissues of rats. (A) Expression of $I L-1 b$ mRNA in SH, TD, TO and TI group of rats. (B) Expression of $I L-6$ mRNA in SH, TD, TO and TI group of rats. (C) Expression of TNF-A mRNA in SH, TD, TO and TI group of rats. (D) Expression of PTGS2 mRNA in SH, TD, TO and TI group of rats. Data was presented as mean $\pm \mathrm{SD}$, each experiment was repeated for three times. ${ }^{*} \mathrm{P}<0.05 \mathrm{vs} \mathrm{SH}$ group of rats. $\# \mathrm{P}<0.05$ vs signal dezocine treatment group. NC: normal group, DT: dezocine treatment group, DO: dezocine treatment combined with RAPGEF3 overexpression group, DI: dezocine treatment combined with RAPGEF3 inhibition group. SH: sham group, TD: dezocine treatment group, TO: dezocine treatment combined with RAPGEF3 overexpression group, TI: dezocine treatment combined with RAPGEF3 inhibition group. 


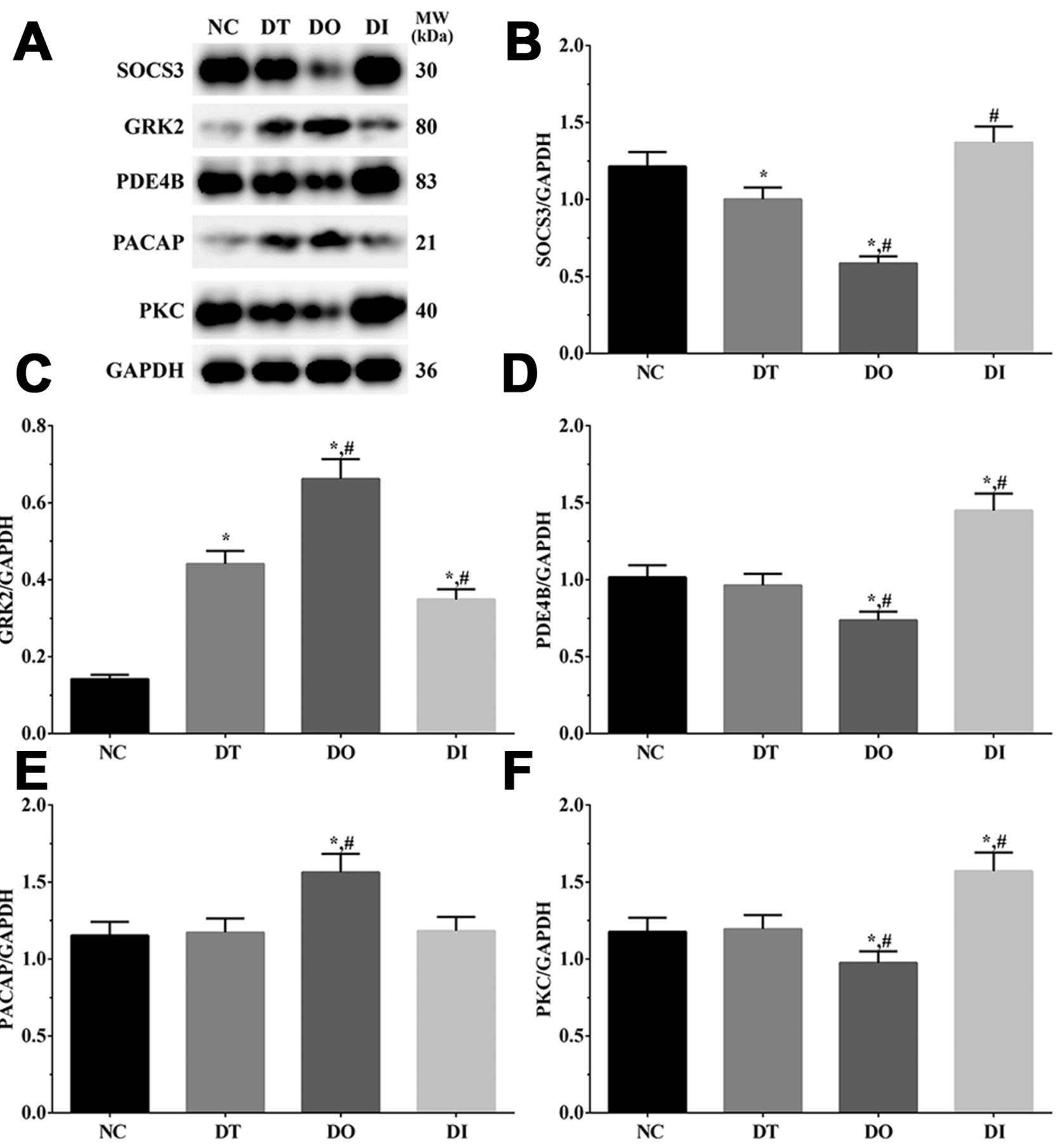

Figure 5 - Detection of neuron protective factors in glial cells. (A) Western blotting analysis of SOCS3, GRK2, PDE4B, PCACP and PKC in glial cells. (B-F) Quantitative analysis of each target protein. Data was presented as mean $\pm \mathrm{SD}$, each experiment was repeated for three times. ${ }^{*} \mathrm{P}<0.05 \mathrm{vs}$ $\mathrm{NC}$ group of cells. $\# \mathrm{P}<0.05$ vs signal dezocine treatment group. NC: normal group, DT: dezocine treatment group, DO: dezocine treatment combined with RAPGEF3 overexpression group, DI: dezocine treatment combined with RAPGEF3 inhibition group. SH: sham group, TD: dezocine treatment group, TO: dezocine treatment combined with RAPGEF3 overexpression group, TI: dezocine treatment combined with RAPGEF3 inhibition group. 


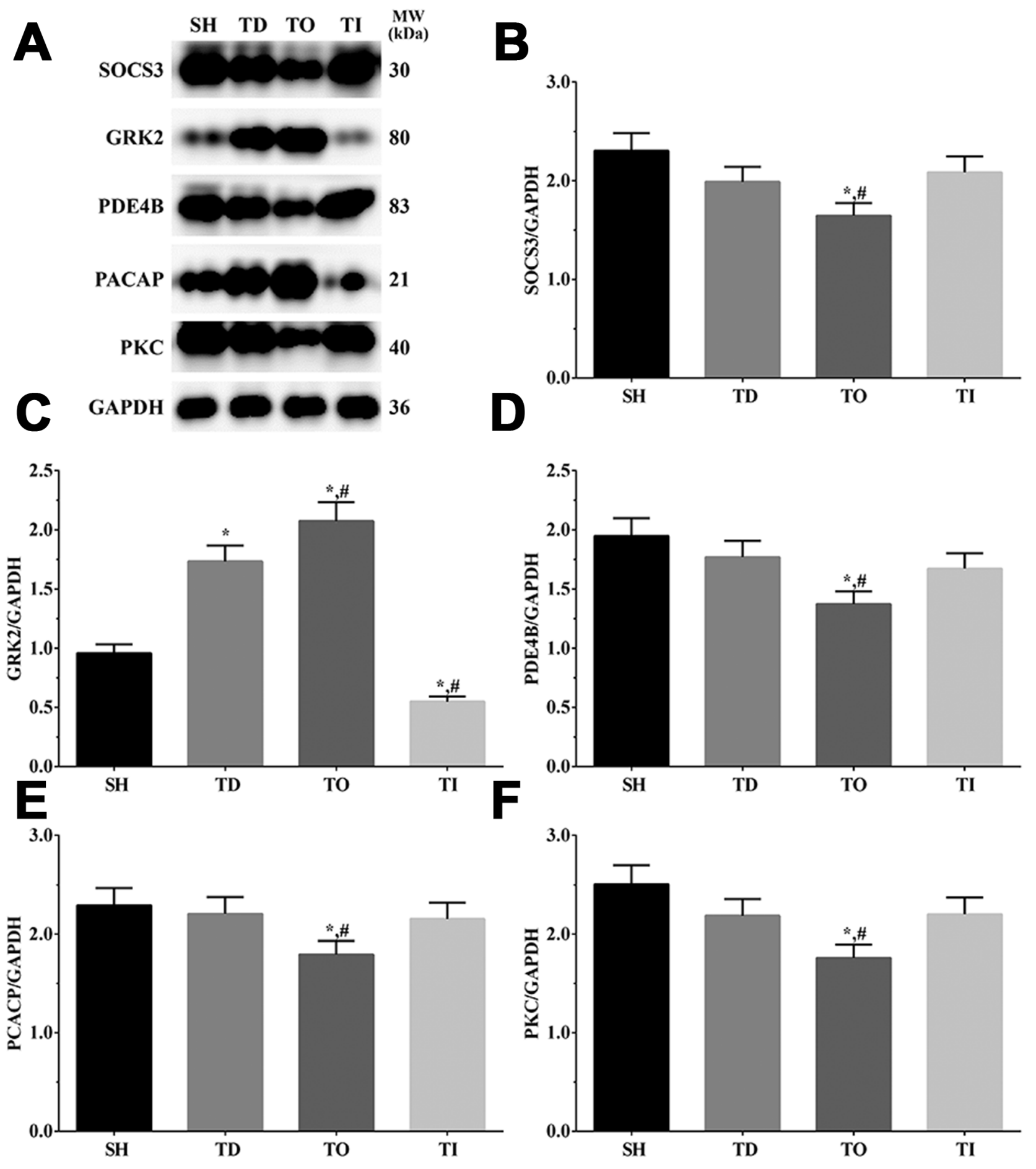

Figure 6 - Detection of neuron protective factors in brain tissue of rats. (A) Western blotting analysis of SOCS3, GRK2, PDE4B, PCACP and PKC in glial cells. (B-F) Quantitative analysis of each target protein. Data was presented as mean $\pm \mathrm{SD}$, each experiment was repeated for three times. ${ }^{*} \mathrm{P}<0.05$ vs SH group of rats. \#P $<0.05$ vs signal dezocine treatment group. NC: normal group, DT: dezocine treatment group, DO: dezocine treatment combined with RAPGEF3 overexpression group, DI: dezocine treatment combined with RAPGEF3 inhibition group. SH: sham group, TD: dezocine treatment group, TO: dezocine treatment combined with RAPGEF3 overexpression group, TI: dezocine treatment combined with RAPGEF3 inhibition group. 


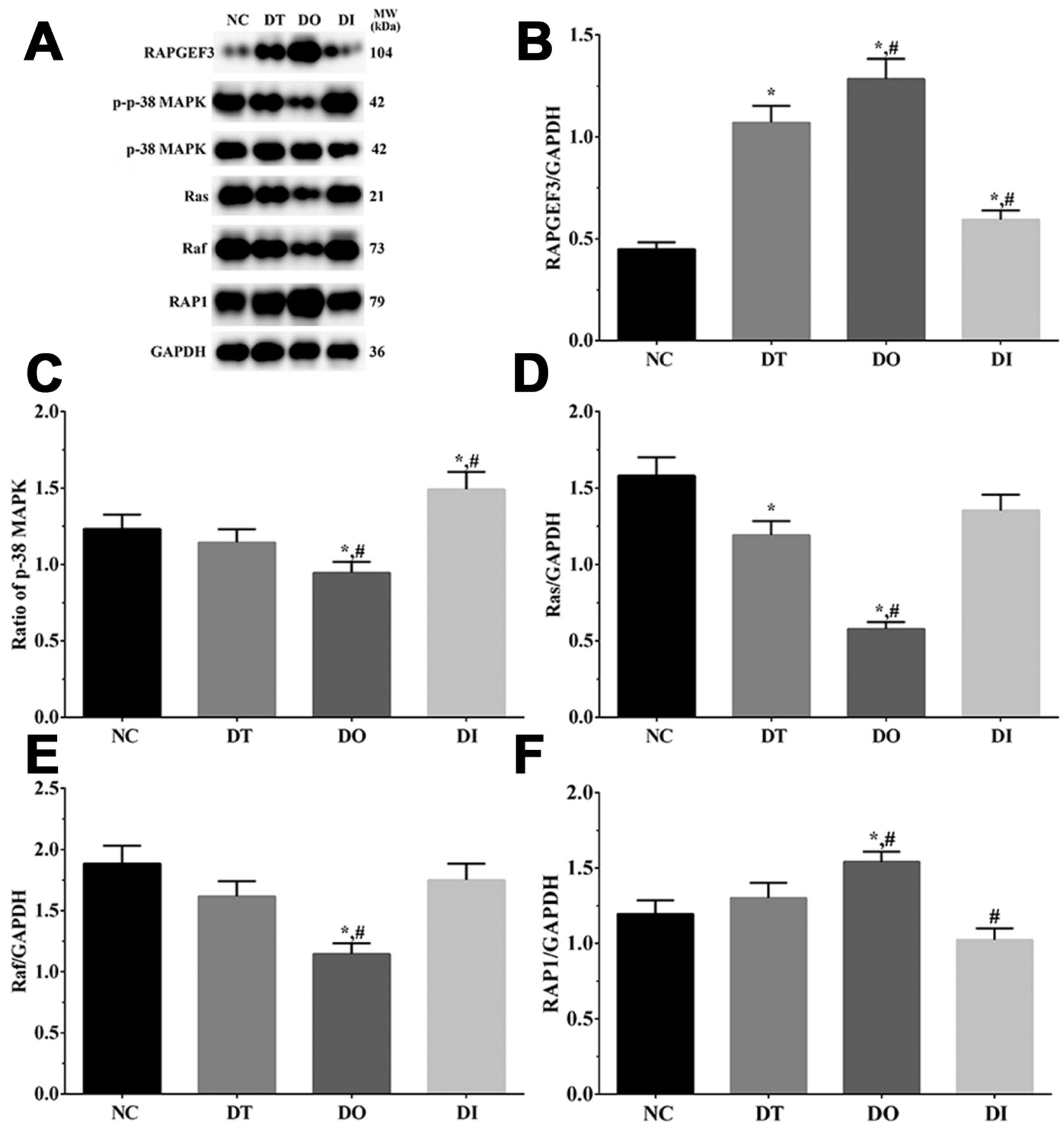

Figure 7 - Activation of Ras/Raf/MAPK in glial cells. (A) Western blotting analysis of RAPGEF3, p-38 MAPK, Ras, Raf and PKC in glial cells. (B-F) Quantitative analysis of each target protein. Data was presented as mean $\pm \mathrm{SD}$, each experiment was repeated for three times. $* \mathrm{P}<0.05$ vs $\mathrm{NC}$ group of cells. \#P $<0.05$ vs signal dezocine treatment group. NC: normal group, DT: dezocine treatment group, DO: dezocine treatment combined with RAPGEF3 overexpression group, DI: dezocine treatment combined with RAPGEF3 inhibition group. SH: sham group, TD: dezocine treatment group, TO: dezocine treatment combined with RAPGEF3 overexpression group, TI: dezocine treatment combined with RAPGEF3 inhibition group. 


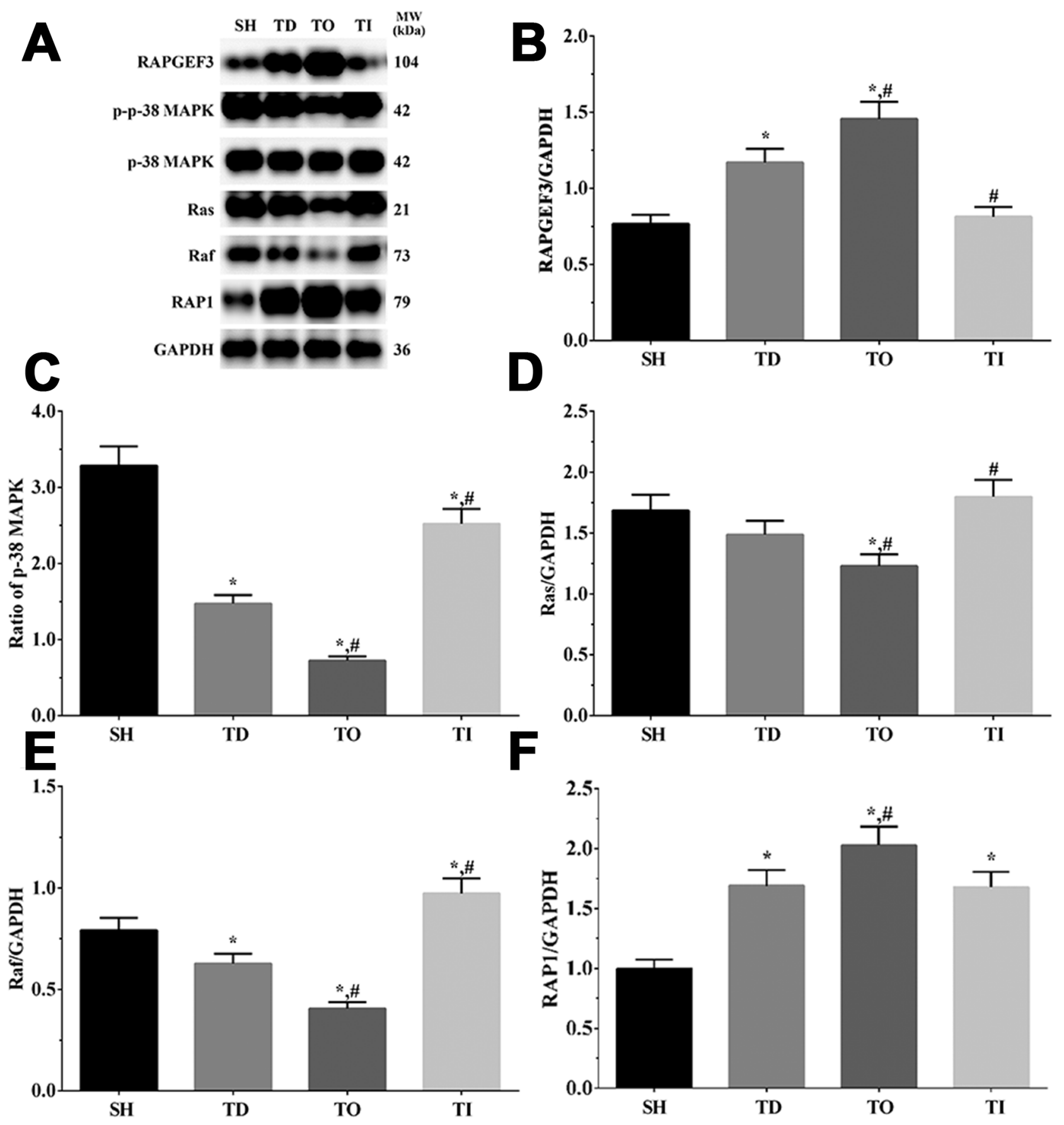

Figure 8 - Activation of Ras/Raf/MAPK in brain tissue of rats. (A) Western blotting analysis of RAPGEF3, p-38 MAPK, Ras, Raf and PKC in glial cells. (B-F) Quantitative analysis of each target protein. Data was presented as mean $\pm \mathrm{SD}$, each experiment was repeated for three times. ${ }^{*} \mathrm{P}<0.05 \mathrm{vs}$ SH group of rats. $\# \mathrm{P}<0.05$ vs signal dezocine treatment group. NC: normal group, DT: dezocine treatment group, DO: dezocine treatment combined with RAPGEF3 overexpression group, DI: dezocine treatment combined with RAPGEF3 inhibition group. SH: sham group, TD: dezocine treatment group, TO: dezocine treatment combined with RAPGEF3 overexpression group, TI: dezocine treatment combined with RAPGEF3 inhibition group. 

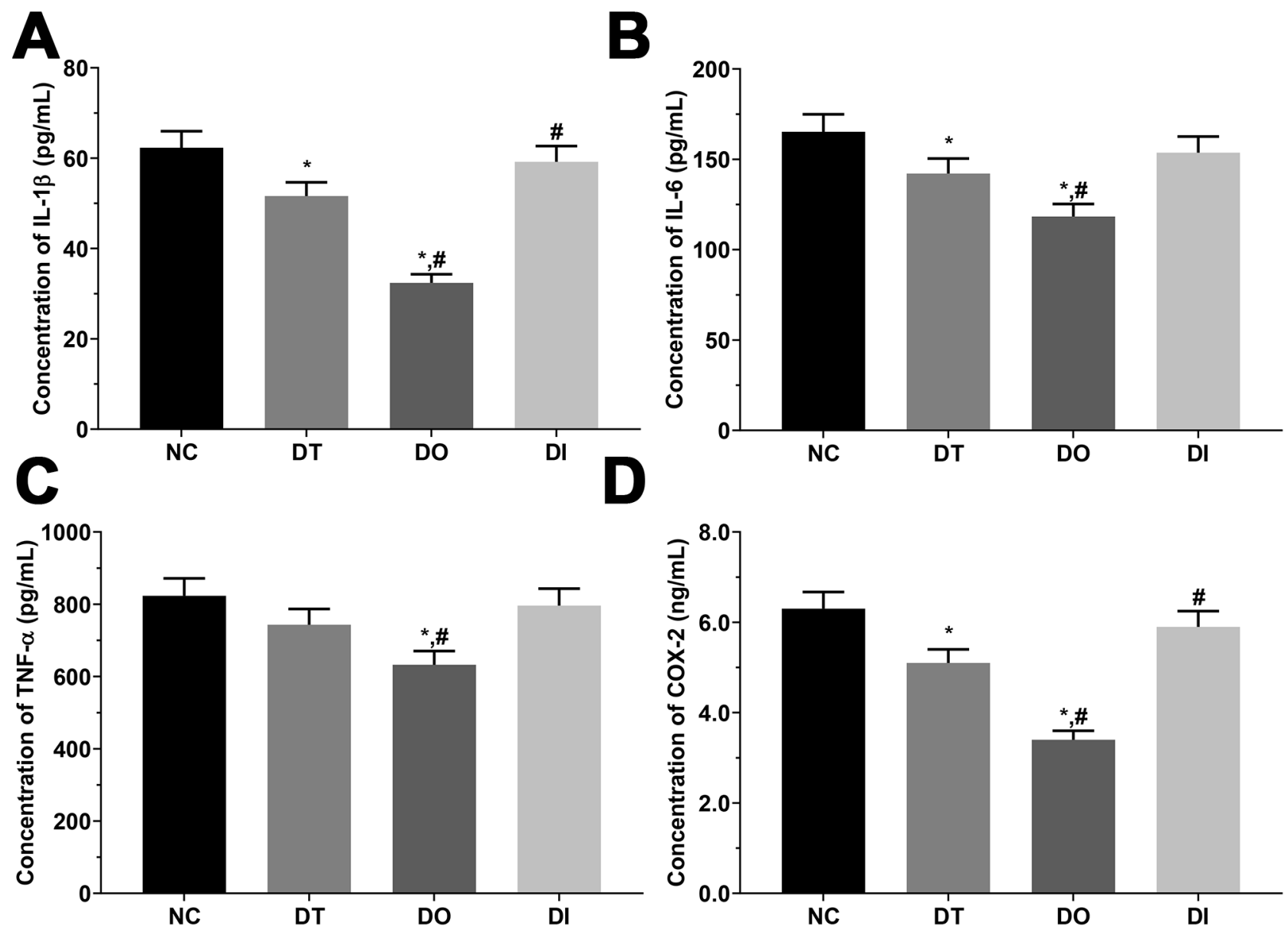

Figure 9 - Concentration of pro-inflammatory factor in cultured medium of glial cells. (A) Concentration of IL-1 $\beta$ mRNA in NC, DT, DO and DI group of glial cells. (B) Concentration of IL- 6 mRNA in NC, DT, DO and DI group of glial cells. (C) Concentration of TNF- $\alpha$ in NC, DT, DO and DI group of glial cells. (D) Concentration of COX-2 in NC, DT, DO and DI group of glial cells. Data was presented as mean \pm SD, each experiment was repeated for three times. ${ }^{*} \mathrm{P}<0.05$ vs $\mathrm{NC}$ group of cells. $\# \mathrm{P}<0.05$ vs signal dezocine treatment group. NC: normal group, DT: dezocine treatment group, DO: dezocine treatment combined with RAPGEF3 overexpression group, DI: dezocine treatment combined with RAPGEF3 inhibition group. SH: sham group, TD: dezocine treatment group, TO: dezocine treatment combined with RAPGEF3 overexpression group, TI: dezocine treatment combined with RAPGEF3 inhibition group.

\section{Discussion}

Injury of the peripheral nervous system induced by traumatic, metabolic, and toxic factors, is the leading cause of neuropathic pain development (Ji et al., 2014). Dezocine has been widely used in clinical trials, and has been found to relieve neuropathic pain with high safety (Ding and White, 1992). A previous study demonstrated that in a CCI mouse model, pain sensitivity could be attenuated by dezocine. This effect presented no signs of tolerance, and the authors surmised that dezocine could be considered an alternative medication for treating NP (Wu et al., 2019). Moreover, a recent study reported that subcutaneous injection of dezocine inhibited NP in patients with cancer in a time-dependent manner (Mao et al., 2020). However, the effect of dezocine in treating NP is not fully clear. Rap guanine nucleotide exchange factor 3 (RAPGEF3) is a regulator of the Ras family, especially Rap1 and Rap2, and is involved in cellular survival, cytokine secretion, and cell migration (Kumar et al., 2017). Based on these characteristics, we hypothesized that RAPGEF3 might contribute to the therapeutic effect of dezocine.
During chronic pain, inflammatory mediators are continuously produced, and pro-inflammatory factors, including TNF- $\alpha$ and IL-1 $\beta$, induce pain through activation and sensitization of nociceptors (Ji et al., 2018). The cyclooxygenase (COX) pathway participates in the precursor substrate of arachidonic acid (AA) and produces prostaglandins (PGs) that play an important role in regulating the inflammatory response. Production of IL- $1 \beta$ induces COX-2 expression, resulting in the production of prostaglandin (PG)E2 (Cheng et al., 2013); furthermore, IL-6 and TNF- $\alpha$ expression is activated, inducing the activation of the inflammatory response process. IL-6 plays a vital role in regulating immune diseases, infections, and metabolic diseases (Rose-John S., 2018. $\mathrm{TNF}-\alpha$ also regulates the inflammatory response process and cytokine activation network (Balkwill, 2009). The expression of COX-2 is also regulated by inflammatory stimuli, such as IL-1, IL-6, and TNF- $\alpha$, further inducing the expression of downstream molecules involved in inflammation and the neoplastic growth process (Song et al., 1998). Here, using qPCR and ELISA, we found that the expression of these pro-inflammatory factors such as IL- $1 \beta$, IL- 6 , TNF- $\alpha$, and 
COX-2, as well as their concentration in serum and culture medium, were decreased, indicating that dezocine treatment decreased the expression and secretion of these cytokines, and overexpression of RAPGEF3 further enhanced this effect, leading to the inhibition of inflammatory response process in NP models, indicating a potential therapeutic effect.

Suppressors of cytokine signaling (SOCS) are known regulators of the Janus kinase (JAK)/ signal transducer and activators of the transcription (STAT) pathway through a negative feedback mechanism (Polizzotto et al., 2000). After damage to neuronal cells, SOCS3 expression is normally upregulated, limiting the function of the ciliary neurotrophic factor (CNTF) on neuron cells, as injection of CNTF in SOCS3 deletion mice induced robust axon regeneration (Smith et al., 2009) and overexpression of SOCS3 diminished the axon regeneration induced by CNTF (Hellström et al., 2011). A previous study found that RAPGEF3 could inhibit the inflammation process induced by IL- 6 through upregulation of SOCS3 (Sands et al., 2006). Another study reported that inhibition of RAPGEF3 leads to the downregulation of PDE4, which in turn inhibits the vascular inflammation process (Michael et al., 2015). Using RAPGEF3 knockdown mice, a recent study showed that RAPGEF3 plays a critical role in regulating chronic inflammatory pain via the regulation of RAPGEF3 phosphorylation through GRK2 (Pooja et al., 2016). Furthermore, it was observed that the interaction between GRK2 and RAPGEF3 contributes to chronic pain in RAPGEF3 knockout mice and that increased GRK2 expression inhibitschronic pain in a chronic inflammatory pain model (Wang et al., 2013). G protein-coupled receptor kinase 2 (GRK2) is a member of the G protein coupled receptor (GPCR) kinase family that regulates the function of inflammatory cells and the secretion of inflammatory cytokines under normal conditions (Tang et al., 2017). Under the activation of inflammatory immune response conditions, expression, and activation of GRK2 are disrupted, resulting in the production of inflammatory cytokines and cell damage (Taguchi et al., 2017). Phosphodiesterase $4 \mathrm{~B}$ (PDE4B) is an enzyme that regulates the intercellular concentration of cAMP (Li et al., 2018). A previous study determined that inhibition of PDE4B expression leads to the accumulation of cAMP, further activation of RAPGEF3 and protection against neuronal apoptosis and inflammatory responses in neuronal cells (Zhong et al., 2019). PACAP was first discovered in 1989 in an ovine hypothalamic extract and has been reported to participate in various developmental processes
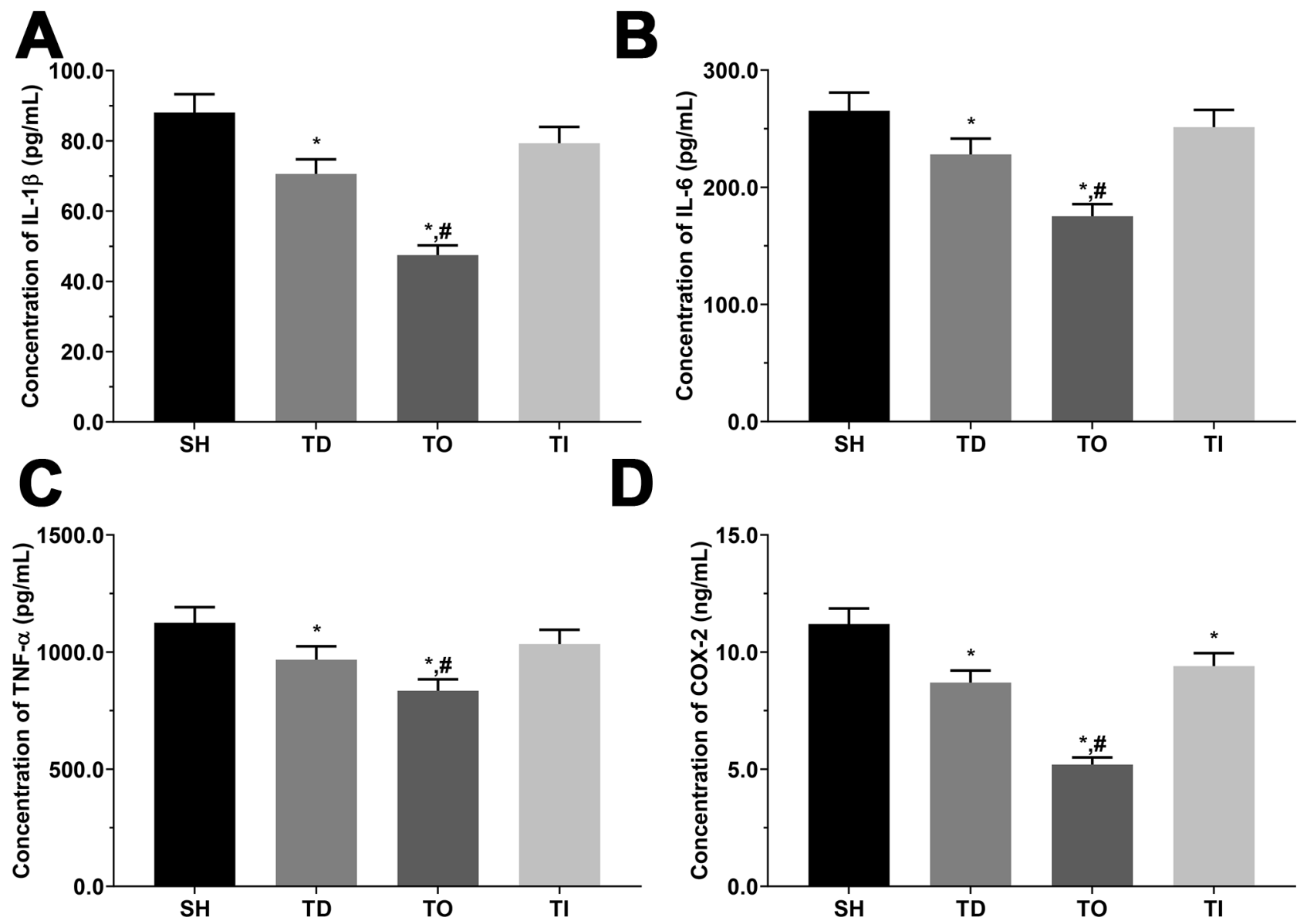

Figure 10 - Concentration of pro-inflammatory factor in serum samples of rats. (A) Concentration of IL-1 $1 \beta$ mRNA in NC, DT, DO and DI group of rats. (B) Concentration of IL-6 mRNA in NC, DT, DO and DI group of rats. (C) Concentration of TNF- $\alpha$ in NC, DT, DO and DI group of rats. (D) Concentration of COX-2 in NC, DT, DO and DI group of rats. Data was presented as mean $\pm \mathrm{SD}$, each experiment was repeated for three times. $* \mathrm{P}<0.05$ vs $\mathrm{SH}$ group of rats. $\# \mathrm{P}<0.05$ vs signal dezocine treatment group. NC: normal group, DT: dezocine treatment group, DO: dezocine treatment combined with RAPGEF3 overexpression group, DI: dezocine treatment combined with RAPGEF3 inhibition group. SH: sham group, TD: dezocine treatment group, TO: dezocine treatment combined with RAPGEF3 overexpression group, TI: dezocine treatment combined with RAPGEF3 inhibition group. 
(Basille et al., 2000). After injury to the nervous system, the expression of several growth factors was elevated, as well as PCACP expression (Johanson et al., 2011), promoting nervous system regeneration. Thus, PCACP is regarded as a neuroprotective factor. It has previously been established that using PKC $\alpha$ antagonists or gene deletion alleviated nociceptive hypersensitivity (Park et al., 2009). Protein kinases, such as MAPK and PKC, are critical nociceptors regulators, resulting in peripheral sensitization induction and maintenance ( $\mathrm{Ji}$ et al., 2009). Further studies have shown that upregulation of PKC $\alpha$ is mediated by direct or indirect activation of the mitogenactivated protein kinase (MAPK) pathway (Yang et al., 2007). Here, we found that the expression of GRK2 and PACAP was increased after overexpression of RAPGEF3, while the expression of SOCS3, PDE4B and PKC was decreased, indicating that overexpression of RAPGEF3 promotes the protective effect of dezocine.

RAPGEF3 is a sensor of the cellular second messenger cAMP and acts as the exchange factor of the Rap and Ras families; increased cAMP expression promotes the translocation of RAPGEF3 to the cellular membrane, further inducing Rap1 activation (Ponsioen et al., 2009). Rap1 is a small molecular weight GTPase that belongs to the subfamily of the Ras family and has been shown to regulate several biological processes, including cellular growth, adhesion, and apoptosis (Takai et al., 2001). Activation of RAP1 induced by RAPGEF3 further leads to the activation of MAPK, resulting in the opening of $\mathrm{K}^{+}$channels to promote cellular proliferation (Han et al., 2006). In addition, a previous study reported that the EPAC1/RAP1 signaling pathway promotes cancer cell proliferation by contributing to the glucose uptake and metabolism ability of cells (Onodera et al., 2014). Here, we observed that RAPGEF3 overexpression leads to an increase in the cellular viability rate, which might be induced by RAP1 and its downstream molecules. The Ras/Raf/MAPK signaling pathway is the central regulator of multiple biochemical signals and regulates numerous cellular processes, including proliferation, differentiation and development (Kawasaki et al., 1998). Accumulating evidence identifies the association between Ras/Raf/MAPK signaling pathways and recovery of neuropsychiatric disorders (Zhong, 2016). A recent study indicated that inhibition of the Ras/Raf/MAPK signaling pathway significantly enhances the migration of neurons in mouse models (Xu et al., 2016b), and also resulted in a greater restoration of dendritic spine density (Kasai et al., 2010), which is essential for synaptic function and recovery of neuronal cells from injury. Furthermore, a previous study indicated that activation of the Ras/MAPK signaling pathway impairs the formation of synapses, and inhibits the development of mature spines (Cao et al., 2013).

\section{Conclusion}

Here, we demonstrated that dezocine treatment combined with overexpression of RAPGEF3 significantly increased the viability of cells, decreased the expression of inflammation-related cytokines at the transcriptional level and the concentration of inflammatory cytokines in cultured medium and serum samples of cells and rats. In contrast, the expression of anti-inflammatory and neuroprotective proteins increased. We further revealed that these effects might be mediated by inhibition of the Ras/Raf/MAPK signaling pathway. Thus, we hypothesized that dezocine treatment combined with RAPGEF3 overexpression might have a therapeutic effect on NP by increasing the proliferation ability of neuronal cells. However, the present results were limited by the small sample size of the rats and the limitation of the research methods. Thus, further experimentation with human samples is required.

\section{Acknowledgments}

This work was supported by Scientific Research Project of Shandong Province (No. 17SSR026).

\section{Conflict of Interest}

The authors declare that there is no conflict of interest that could be perceived as prejudicial to the impartiality of the reported research.

\section{Author Contributions}

XL conducted cell culture, vector construction, nucleic acid experiment and protein experiments and wrote the manuscript, LS performed animal housing and behavior tests, XJM performed animal housing, model construction and ELISA experiments and analyzed the data, YL, HH and YSX performed the statistical analysis and collected the samples. WY conceived the study and revised the manuscript.

\section{References}

Kroth A, Santos MDCQ, Borella da Silva TC, Santos Silveira EM and Partata WA (2020) Aqueous leaf extract from Luehea divaricata Mart. modulates oxidative stress markers in the spinal cord of rats with neuropathic pain. J Ethnopharmacol 268:113674.

Balkwill F (2009) Tumor necrosis factor and cancer. Nat Rev Cancer 9:361-371.

Basille M, Vaudry D, Coulouarn Y, Jegou S, Lihrmann I, Fournier A, Vaudry H and Gonzalez B (2000) Comparative distribution of pituitary adenylate cyclase-activating polypeptide (PACAP) binding sites and PACAP receptor mRNAs in the rat brain during development. J Comp Neurol 425:495-509.

Bouhassira D (2019) Neuropathic pain: Definition, assessment and epidemiology. Rev Neurol (Paris) 175:16-25.

Bos JL (2006) Epac proteins: multi-purpose cAMP targets. Trends Biochem Sci 31:680-686.

Bramanti V, Grasso S, Tibullo D, Giallongo C, Raciti G, Viola M and Roberto A (2015) Modulation of extracellular signal-related kinase, cyclin D1, glial fibrillary acidic protein, and vimentin expression in estradiol-pretreated astrocyte cultures treated with competence and progression growth factors. J Neurosci Res 93:1378-1387.

Cao FJ, Zhang X, Liu T, Li XW, Malik M and Feng SQ (2013) Up-regulation of Ras/Raf/ERK1/2 signaling in the spinal cord impairs neural cell migration, neurogenesis, synapse formation, and dendritic spine development. Chin Med J (Engl) 126:3879-3885.

Carswell EA, Old LJ, Kassel RL, Green S, Fiore N and Williamson B (1975) An endotoxin-induced serum factor that causes necrosis of tumors. Proc Natl Acad Sci U S A 72:3666-3670.

Chaplan SR, Bach FW, Pogrel JW, Chung JM and Yaksh TL (1994) Quantitative assessment of tactile allodynia in the rat paw. J Neurosci Methods 53:55-63. 
Cheng W, Wu D, Zuo Q, Wang Z and Fan W (2013) Ginsenoside Rb1 prevents interleukin-1 beta induced inflammation and apoptosis in human articular chondrocytes. Int Orthop 37:2065-2070.

Ding Y and White PF (1992) Comparative effects of ketorolac, dezocine, and fentanyl as adjuvants during outpatient anesthesia. Anesth Analg 75:566-571.

Ding Y, Yao P, Hong T, Han Z, Zhao B and Chen W (2017) The NO-cGMP-PKG signal transduction pathway is involved in the analgesic effect of early hyperbaric oxygen treatment of neuropathic pain. J Headache Pain 18:51.

Grimholt RM, Urdal P, Klingenberg O and Piehler AP (2013) Rapid and reliable detection of $\alpha$-globin copy number variations by quantitative real-time PCR. J Mol Diagn 15:642-651.

Han J, Lim CJ, Watanabe N, Soriani A, Ratnikov B, Calderwood DA, Wilma PM, Esther ML, Vassiliki AB, Sanford JS and Mark HG (2006) Reconstructing and deconstructing agonist-induced activation of integrin $\alpha \operatorname{IIb} \beta 3$. Curr Biol 16:1796-1806.

Han L, Yuan B, Shimada R, Hayashi H, Si N, Zhao HY, Baolin B and Norio T (2018) Cytocidal effects of arenobufagin and hellebrigenin, two active bufadienolide compounds, against human glioblastoma cell Line U-87. Int J Oncol 53:2488-2502.

Ji RR, Gereau RW, Malcangio M and Strichartz GR (2009) MAP kinase and pain. Brain Res Rev 60:135-148.

Ji RR, Xu ZZ and Gao YJ(2014) Emerging targets in neuroinflammationdriven chronic pain. Nat Rev Drug Discov 13:533-548.

Ji RR, Nackley A, Huh Y, Terrando N and Maixner W (2018) Neuroinflammation and central sensitization in chronic and widespread pain. Anesthesiology 129:343-366.

Johanson C, Stopa E, Baird A and Sharma H (2011) Traumatic brain injury and recovery mechanisms: Peptide modulation of periventricular neurogenic regions by the choroid plexus-CSF nexus. J Neural Transm (Vienna) 118:115-133.

Kasai H, Fukuda M, Watanabe S, Hayashi-Takagi A and Noguchi J (2010) Structural dynamics of dendritic spines in memory and cognition. Trends Neurosci 33:121-129.

Kawasaki H, Springett GM, Mochizuki N, Toki S, Nakaya M, Matsuda M, Housman DE and Graybiel AM (1998) A family of cAMP-binding proteins that directly activate Rap1. Science 282:2275-2279.

Kumar N, Gupta S, Dabral S, Singh S and Sehrawat S (2017) Role of exchange protein directly activated by cAMP (EPAC1) in breast cancer cell migration and apoptosis. Mol Cell Biochem 430:115-125.

Li H, Zuo J and Tang W (2018) Phosphodiesterase-4 inhibitors for the treatment of inflammatory diseases. Front Pharmacol 9:1048.

Li NN, Huang YQ, Huang LE, Guo SH, Shen MR, Guo CL, Zhu SM and Yao YX (2017) Dezocine antagonizes morphine analgesia upon simultaneous administration in rodent models of acute nociception. Pain Physician 20:E401-E409.

Liu C, Zhang L, Liu H and Cheng K (2017) Delivery strategies of the CRISPR-Cas9 gene-editing system for therapeutic applications. J Control Release 266:17-26.

Mao XF, Ahsan MZ, Apryani E, Tang XQ, Zhao MJ, Li XY and Wang YX (2020) Dual $\mu$-opioid receptor and norepinephrine reuptake mechanisms contribute to dezocine- and tapentadol-induced mechanical antiallodynia in cancer pain. Eur J Pharmacol 876:173062.

Michael L, Florian K, Anna M, Pathricia VT, Sebastian D, Judith M, Robert S, Katharina H, Elizabeth BE, Dennis B, Nikolaus M and Hannes MF (2015) PDE4 inhibition reduces neointima formation and inhibits VCAM-1 expression and histone methylation in an Epac-dependent manner. J Mol Cell Cardiol 81:23-33.

Onodera Y, Nam J and Bissell MJ (2014) Increased sugar uptake promotes oncogenesis via EPAC/RAP1 and O-GlcNAc pathways. J Clin Invest 124:367-384.
Pan P, Huang SS, Shen SR, Lu CE, Qin YB, Zhang JL and Cao S (2019) Role of p120 catenin in Epac1-induced chronic postsurgical pain in rats. Pain Res Manag 2019:9017931.

Pandit UA, Kothary SP and Pandit SK (1986) Intravenous dezocine for postoperative pain: a double-blind, placebo-controlled comparison with morphine. J Clin Pharmacol 26:275-280.

Park JS, Voitenko N, Petralia RS, Guan X, Xu JT, Steinberg JP, Kogo T, Andrij S, Olga K, Richard LH and Tao YX (2009) Persistent infammation induces GluR2 internalization via NMDA receptor-triggered PKC activation in dorsal horn neurons. J Neurosci 29:3206-3219.

Ponsioen B, Gloerich M, Ritsma L, Rehmann H, Bos JL and Jalink K (2009) Direct spatial control of Epac1 by cyclic AMP. Mol Cell Biol 29:2521-2531.

Polizzotto MN, Bartlett PF and Turnley AM (2000) Expression of "suppressor of cytokine signalling" (SOCS) genes in the developing and adult mouse nervous system. J Comp Neurol 423:348-358.

Pooja S, Huo XJ, Niels E, Susana RB, Faiza B, Mei FC, Zhu YM, Cheng CD, Hawke D, Mayor Jr F, Murga C, Heijnen CJ and Kavelaars A (2016) Critical role for Epac1 in inflammatory pain controlled by GRK2-mediated phosphorylation of Epac1. Proc Natl Acad Sci U S A 113:3036-3041.

Sands WA, Woolson HD, Milne GR, Rutherford C and Palmer TM (2006) Exchange protein activated by cyclic AMP (Epac)mediated induction of suppressor of cytokine signaling 3 (SOCS-3) in vascular endothelial cells. Mol Cell Biol 26:63336346.

Smith PD, Sun F, Park KK, Cai B, Wang C, Kuwako K, Irene MC, Lauren C and He ZG (2009) SOCS3 deletion promotes optic nerve regeneration in vivo. Neuron 64:617-623.

Song C, Lin A, Bonaccorso S, Heide C, Verkerk R, Kenis G, Bosmans E, Scharpe S, Whelan A, Cosyns P et al. (1998) The inflammatory response system and the availability of plasma tryptophan in patients with primary sleep disorders and major depression. J Affect Disord 49:211-219.

Taguchi K, Hida M, Hasegawa M, Narimatsu H, Matsumoto T and Kobayashi T (2017) Suppression of GRK2 expression reduces endothelial dysfunction by restoring glucose homeostasis. Sci Rep 7:8436.

Takai Y, Sasaki T and Matozaki T (2001) Small GTP-binding proteins. Physiol Rev 81:153-208.

Tang J, Guo Z, Zhang Y, Bai B and Zhu WH (2017) Rational design of a fast and selective near-infrared fluorescent probe for targeted monitoring of endogenous nitric oxide. Chem Commun (Camb) 53:10520-10523.

Walker EA, Tiano MJ, Benyas SI, Dykstra LA and Picker MJ (1999) Naltrexone and beta-funaltrexamine antagonism of the antinociceptive and response rate-decreasing effects of morphine, dezocine, and d-prop-oxyphene. Psychopharmacology 144:45-53.

Wang H, Heijnen CJ, van Velthoven CTJ, Willemen HLDM, Ishikawa Y, Zhang X, Anil KS, Anne V, Niels E and Annemieke K (2013) Balancing GRK2 and EPAC1 levels prevents and relieves chronic pain. J Clin Invest 123:5023-5034.

Wu FX, Babazada H, Gao H, Huang XP, Xi CH, Chen CH, Xi J, Yu WF and Liu RY (2019) Dezocine alleviates morphine-induced dependence in rats. Anesth Analg 128:1328-1335.

Xu BB, Zhao XL and Xu GP (2016a) Clinical study of anesthetization by dezocine combined with propofol for indolent colonoscopy. World J Gastroenterol 22:5609-5615.

Xu D, Cao F, Sun S, Liu T and Feng S (2016b) Inhibition of the Ras/Raf/ERK1/2 signaling pathway restores cultured spinal cord-injured neuronal migration, adhesion, and dendritic spine development. Neurochem Res 41:2086-2096. 
Yang HQ, Pan J, Ba MW, Sun ZK, Ma GZ, Lu GQ, Xiao Q and Chen SD (2007) New protein kinase $C$ activator regulates amyloid precursor protein processing in vitro by increasing $\alpha$-secretase activity. Eur J Neurosci 26:381-391.

Yokoyama U, Minamisawa S, Quan H, Akaike T, Jin M, Otsu K, Ulucan C, Wang X, Baljinnyam E, Takaoka M, Sata M and Ishikawa Y (2008) Epac1 is upregulated during neointima formation and promotes vascular smooth muscle cell migration. Am J Physiol Heart Circ Physiol 295:H1547-1555.

Zhong J (2016) RAS and downstream RAF-MEK and PI3K-AKT signaling in neuronal development, function and dysfunction. Biol Chem 397:215-222.
Zhang GF, Guo J, Qiu LL, Li SM, Zheng M, Xia JY and Yang JJ (2019) Effects of dezocine for the prevention of postoperative catheter-related bladder discomfort: a prospective randomized trial. Drug Des Devel Ther 13:1281-1288.

Associate Editor: Daisy Maria Fávero Salvadori

License information: This is an open-access article distributed under the terms of the Creative Commons Attribution License (type CC-BY), which permits unrestricted use, distribution and reproduction in any medium, provided the original article is properly cited.Obsed non ta que iusquam pernihilina, idicaes pere cultum sa publiciviris horidest incles bonihinate, cerum sigilicotam que aciem ignat, nihiliisquam mus hoctus, quid auceriorio, caverit, et que in sil hocusquidem es opotarit. Hus hales in dea terum sit plis, nenat issulis sederi 